\title{
Mixed Batch and Transactional Workloads for Cloud Computing Jobs
}

\author{
M. Kalai Selvi \\ (ME) PG Scholar \\ Karpagam Academy of Higher Education \\ Coimbatore-21
}

\author{
B. Arunkumar ME, PhD \\ Assistant Professor \\ Karpagam Academy Of Higher Education \\ Coimbatore-21
}

\begin{abstract}
In this mixed batch and transactional workloads for cloud computing jobs we implemented a technique that manages a long running jobs and OLTP it contains mixed workloads of all the types like word, video and image. In this process job scheduler plays an important role, it is assigned for managing workloads and also is an application for controlling non viewing or unattended background program process or execution. Our proposed and implementation process is, it allows miscellaneous workloads are to be collected on any one of the server machine so that we can able to reduce the decision making process of resource allocation. In the previous paper the workloads of any type to be allotted to nearest server it leads long time to complete the process and also minimum bandwidth process is also delayed to overcome this Problem we analyses certain process that first the workloads to be analyzed and it be allotted for the server using the resource allocation and scheduler. We reveal that our technique maximizes mixed workload performance while providing service demarcation based on complex performance goals.
\end{abstract}

\section{General terms}

OLTP, TCP connection, cloud, VC and BL

\section{Keywords}

Cloud Computing, Job scheduler, Resource allocation Virtualization and Map reduce

\section{INTRODUCTION}

Cloud is a one of the growing technology it reaches a high position in the market for many things, it needs an high network connectivity for a usage of cloud computing there are many users focuses on the cloud use age because it is an low cost process that is pay for use method the services will be provided by the demands of users it has a different services like software as a service platform as a service and the infrastructure as a service, users for cloud computing is increased because the benefits of cloud in terms of cost unlimited storage.

Based on the cloud user request will provide the connection to the user by giving the access, in the public cloud services we need more number of $\mathrm{VM} s$ to access the large number of request we have to allocate the VM s for the users to access the cloud. And VMs is connected by the TCP connection for the data transfer. Bandwidth for each and every process is based on the competing TCP connection concern of the process, the main reason for more users in cloud is it is an fast and efficient process and also pay for use method but the VM and the bandwidth TCP flows are high we cannot able to guarantee the network process if the small process occupies the large bandwidth it leads a time consumption of the work. And also we have to wait for the long time for a completion of a small job even though it is small process we have to wait for a long time to complete a process.
It is a very big problem in the cloud we are doing the many research work related to map reduce scheduling but the thing is we are allocating the job to the cloud or to the VMs for an process but the we cannot able to guarantee that they should give a result in a allocated time or within the time limit there are two main problems are there in the cloud that is bandwidth fragmentation and the high bandwidth is allocated to the small size of the job, these fragmentation can possible by network allotment or the high bandwidth allotment we are proposed some method for an bandwidth guarantee that is the mixed batch allocation for cloud computing jobs in the dead line cloud computing. It is a very big process in the cloud computing to analysis the VMs and and improves the utilization of a bandwidth

If we want a minimum bandwidth guarantees. we have to focused on certain things we have to analysis the given request and we have to split based on the workloads or the size then we have to allocate a job to the VMs for an process the process allocation or resources allocation of a job is always in the nearest server or nearest VMs but it leads to the time consumption and the small band width or small size process have to wait for the long time this is an common mistake we faced in the cloud, users are mainly focused in the fast and clear output if we are focusing on the nearest server the process for small work or small bandwidth users are affected for the dallying of process.

In this mixed batch and transactional workloads for cloud computing jobs we implemented a technique that manages a cloud computing jobs by the deadline computing this can be done in by creating the new interface between the deadline cloud and the provider first we have to analysis the given job in the and allocate the proper bandwidth to the VMs or in the cloud based on the request analysis we have spit the process based on the bandwidth or size and also we have to analysis the VMs and the cloud setup then have to allocate a job to the correct VMs based on the size of an document based on this process we can eliminate the time consuming process and will guarantee the job to the users. In the many places in the market are using the cloud computing for an easy process the places like Education institution for practical purpose and industries for an data storages and many business peoples are using this cloud for their usage this is to be an sensitive part because dates should to maintained secure basses that should be retrieved in case of any damages in the VM s are the cloud allocation so the process should be a fast and have give a guarantee to the process while the request is raised.

For the Deadline cloud we are using the novel algorithm for a resources allocation during the peak time that is if the more persons are requested to the same content or requested for different process the VM utilization is high to manage these type of process have to do the allocation for the free rooms that can be done by this novel algorithm the process allocated to the different VMs for process once the process is completed with a 
deadline source the room will be kept free for next allocation of job. It will maintain the deadline time process.

\section{PROBLEM STATEMENT}

Problem analysis is used to find the causes of a positive or a negative deviation. In the existing system, the user request is not analyzed properly to allocate the suitable server. Diverse types of workload require diverse control contrivances for management. Diverse workloads are allotted manually to the server. It will create a problem of overloading. The resources are not utilized properly. Workload takes important consideration while doing the large scale application. There is no scheduling strategy in the existing system to reduce the overhead. To avoid this overhead problem, the new plan is implemented. Existing system has some problems that are Account only system work load and perform based on it. Incoming job nature is not analyzed (i.e. job nature and workload) before send to the server for request.

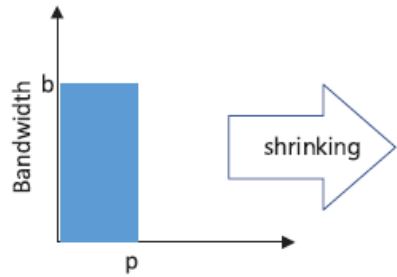

Job running duration

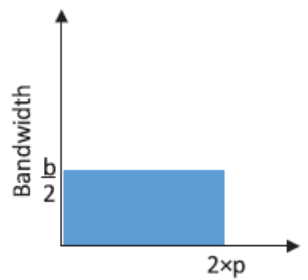

Job running duration
The process can do the asset for the business needs in the industries the process of each system is maintained and the relationship for each process is and the component is linked and the located in the process the design process of an cloud is satisfies the needs of an algorithm like resources allocation and novel algorithm.

\section{RELATED WORKS}

Cloud computing Services, commonly non-trusted tenant set up their services in mutual datacenter infrastructure. Each renter consist of a set of one or more Virtual machines (VMs) placed on one or more physical machines. Cloud environments have a strong requirement to impose recital separation among tenant that share a datacenter, but currently mechanisms are lacking to provide recital separation for datacenter network I/O resources.

Management of network bandwidth is dynamic to handle the high and upward range of service workloads that stress local area network resource in the datacenter. Data intensive application on scalable framework like map reduce can be a highly network intensive. And also upcoming datacenter will merge the traditional messaging traffic with the current network storage traffic into a single unit of datacenter fabric, using file storage new network principles and spread storage space.

In this mixed batch and transactional workloads for cloud computing jobs possessions that multi-tenant network method will satisfied or to meet the practical needs of both the persons that is clouds users and cloud datacenter providers in this previous techniques fails to cover these requirements but in these we statement on our noteworthy development in erection an I/O virtualization control system called gate keeper, this gate keeper will full fill the needs of both user and the provider this can be work in complex applications(this method will work for large scale). That need per-VM state to be maintained at each switch are unfeasible if the need to manage a large amount of state at high speed sanitizes the switches excessively luxurious for cloud computing communications.

\section{CHALLENGES AND CONTRIBUTIONS}

\subsection{Profiling Relax Index}

In this the allocation method and parameter for profiling relaxes index $\mathrm{G}$, it is used to mask the profiling vagueness. In this the value is differ from 0 to 12 percentage in the previous method the limit is below 12 percentage, then the anther parameters is set as default we can keep back more resources than requested for job in each case, we do not insert the in information necessary resource to sprint the job. beneath dissimilar settings we calculate the four metrics and which has been show in the Fig. 4.

It is noticed with the intention of the method of profiling slow down guide has a minimum number of slight collision to all the for parameter or metrics for an example When $G$ is increased from 0 to 12 percentage, the value of victorious job dropped by 2.8 percentage and providers drop by 4 percentage. The drops on a VM and band with consumption are between 3.5 and 2 percentage in that order. The main reason for this is we always unadventurously keep additional resource for an aimed job, if the work is completed the resources is allocated to the additional jobs when aim job is completed prior than the anticipation. In this simulation method we set $G$ value as $1 / 4$ $12 \%$ in worst case for deadline cloud allocation.

\subsection{Deadline annexes Ratio}

The maximum predictable deadline resources additional room ratio $\mathrm{R}$ is the large space to cloud provider for reshaping the request for this the location might depending on request type, then $\mathrm{R}$ is vary from 0 to 20 and also appreciate the universal collision as of the Diagram we can see the deadline additional room ration $\mathrm{R}$ has nearly there is no collision on $\mathrm{VC}$, in the process VC is not judge the job dead line in any way For BL and Deadline cloud.

This dead line cloud completes the more job with the use of value R. the main gain for this process is from large room for both sliding and bandwidth process, but for BL the higher level of job is completed before a longer dead line if the value of $R$ $1 / 41$ then there is no room for either sliding or bandwidth scaling,

the method of $\mathrm{VC}$ also the same process but BL performs the most awful amongst the three, as there is no bandwidth assurance and also a few acknowledged job cannot completed earlier than the Deadline time. In our VC process give bandwidth assurance and the owed jobs for VC can be finished contained by the time limit or deadline, but it can accept very fewer jobs than the Deadline cloud if the $\mathrm{R}$ value is high as 20 , the deadline cloud complete its 40 to 80 percentage of supplementary jobs than the VC and BL.

For an better distribution algorithm the process utilize the proper slots of VM. We are posted the result for the process for the above metric and both VC and BL are not exaggerated by the time limit annex relation.

$\mathrm{VM}$ is very long consumption of job since of the available VM slots (nearly 100 percentages) in spite of the bandwidth demands. Deadline cloud performs superior than the VC, since it accept the additional jobs. The rate of $\mathrm{R}$ is also higher because the supplementary bandwidth scaling prolong the VM profession. If the Deadline cloud extension ration is 20 , the Deadline cloud utilize the almost three times that of VC.

$\mathrm{VC}$ and BL has nearly the same result than the dead line cloud because it leads to bandwidth waste while opposition base 
bandwidth process distribution in BL might origin bandwidth dissipate if the server links are in the excessive condition we also focused on the interchange forwarded among the VMs as an additional metric but the lagging of breathing space we could not able to consider the graph. Dead line cloud performs better than the VM in the process of metric.

It can be possible because it contained more VM allocation by bandwidth scaling and then traffic is forwarded to VMs positioned in the identical bodily server system in cloud.

\subsection{Requested Bandwidth per VM}

Amount of bandwidth used for each VM is one more vital restriction in the resource demand as it indicates the steadiness connecting the VM resources and network assets. The resources are balanced by adopting the technique of bandwidth scaling in the Dead line Cloud due to which it has more advantage.

The comparison between the four metrics with the wideranging bandwidth is depicted in figure 6 . As the demand for the bandwidth increases, the consumption of the resource by $\mathrm{VM}$ also increases and as a result, the proportion of the victorious jobs will be lesser that be depicted in the Fig. 6a. it becomes increasingly complex to assign VMs for jobs with advanced bandwidth requirements in case of Dead line Cloud and VC.

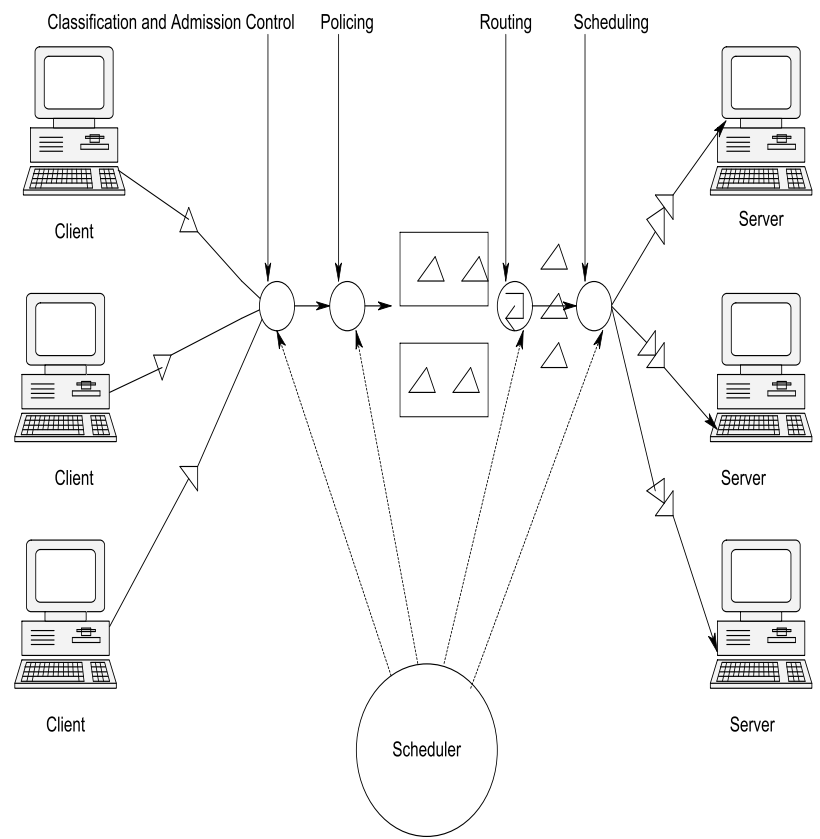

The probability of the violation of the deadline by a job becomes higher for $\mathrm{BL}$ as the job running duration also increases.

In case of Dead line Cloud and VC, as the VM depletes supplementary bandwidth, the on the whole VM consumption becomes lesser and hence fewer jobs are accepted. But, the decrease is not linear in case of Dead line Cloud. As the bandwidth increases more than $450 \mathrm{Mbps}$, the VM utilization goes up even more in Dead line Cloud as the bandwidth scaling and extended VM tenancy increases even more.

From Fig. 6c, one can see that the performance of Dead line Cloud is better than that of the additional two while estimate using the attendant link consumption. As the bandwidth increases, the server link utilization in Dead line. If the cloud is shrinking the band width for VM s is indirectly telling that the process will not be meeting the deadline.

In the process of earnings of Dead line Cloud will be 926 percent more than the $\mathrm{VC}$ and the $\mathrm{BL}$, respectively when the bandwidth requirement is as low as 50 Mbps. The gap increases even more when the bandwidth requirement is as high as $500 \mathrm{Mbps}$,

\section{METHODOLOGY OVERVIEW 5.1 Batch Processing}

Batch processing is yet another way of probable model of job arrival, wherein all the jobs simultaneously arrive at $\mathrm{t} 1 / 40$. We have to study the source of benefits, the Dead line Cloud reserve distribution is reviewed in terms of descending only and by bandwidth scale only The batch is defined as the proportion of jobs meeting deadlines in opposition to the number of jobs. In batch processing, more jobs can be accepted by the Dead line Cloud as compared to the VC and BL, in particular when the number of works amount in the batch is large. Also, the time descending and bandwidth scaling in Dead line Cloud assists in acceptance of more number of jobs.

Fig. $9 \mathrm{~b}$ depicts the income of the cloud supplier. As the time descending and bandwidth scaling helps to recognize more number of jobs to Dead line Cloud, the income too increases. But this is not so in case of the $\mathrm{VC}$ and BL. The reason being that even though more jobs are taken, due to the usage of instantaneous cloud resource, the profit earned is less. In the consignment dealing out, time descending in Dead line Cloud share contribute extra bandwidth scaling in or dealing with the peak demand. But by bandwidth scaling only, Dead line Cloud can also earn 176 percent more than the VC and BL.

\subsection{Summary of the imitation Results}

The chief observations can be summarized below.The efficiency of Dead line Cloud is not extremely perceptive to the assortment of the profile rest index, even if BL has the maximum VM consumption; it is not a completion to complete a job in the given time.

Dead line Cloud has a great deal advanced VM consumption than $\mathrm{VC}$ and has the more utilization of network in the cloud among the three, compared with all the process we are stated that compared to $\mathrm{VC}$ deadline cloud will complete more job than the VC and BL and waiting for an another job for an process the work completion ration is twice the other ones and also it accepts the half percentage that is 50 percentage of cloud provider so that we finalized our process by telling the status of Deadline cloud the dead line cloud has a superior performance under the dead line extension ratio, and also it leads to the higher job advent rate. 

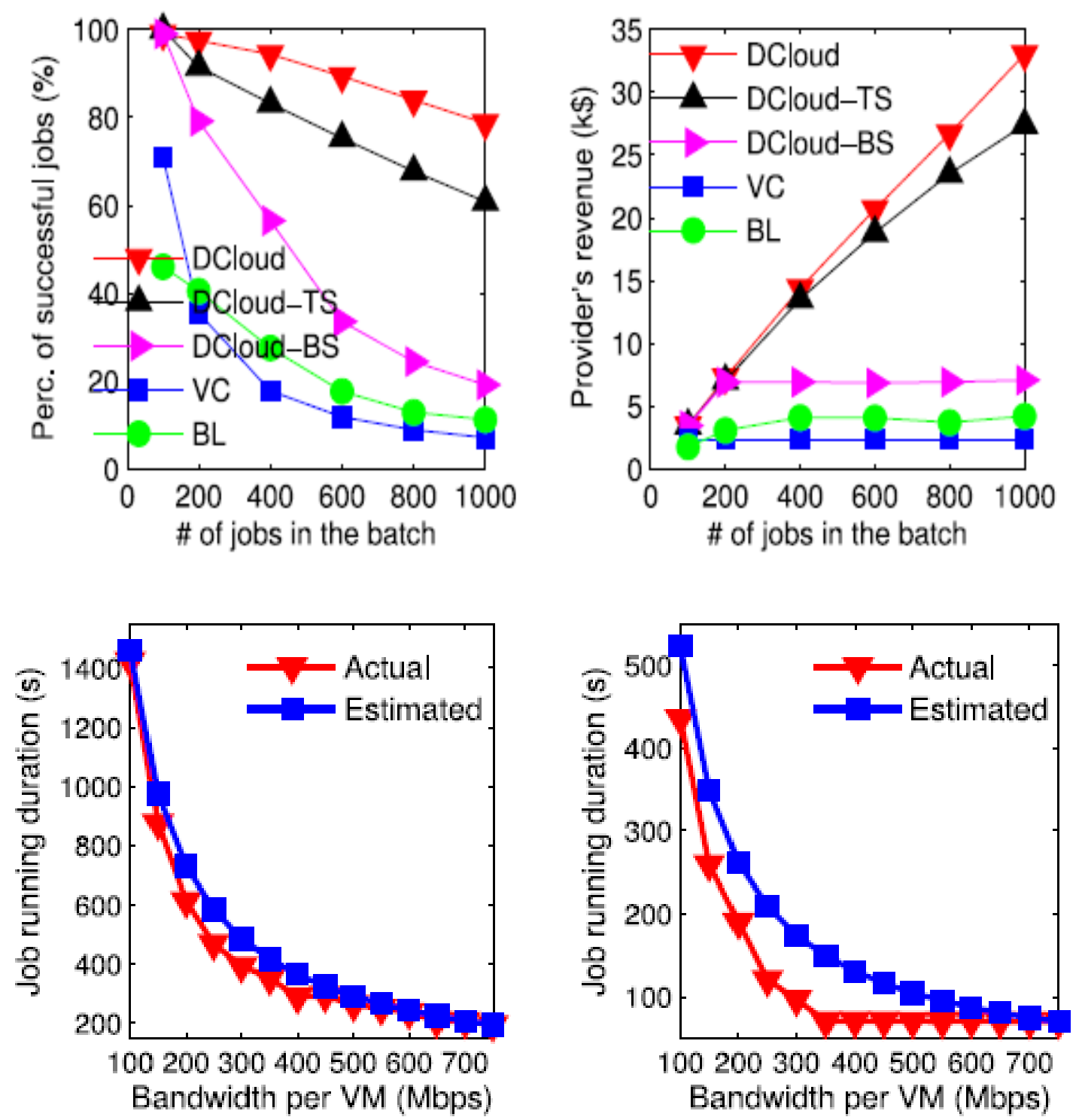

(a) Network-limited application
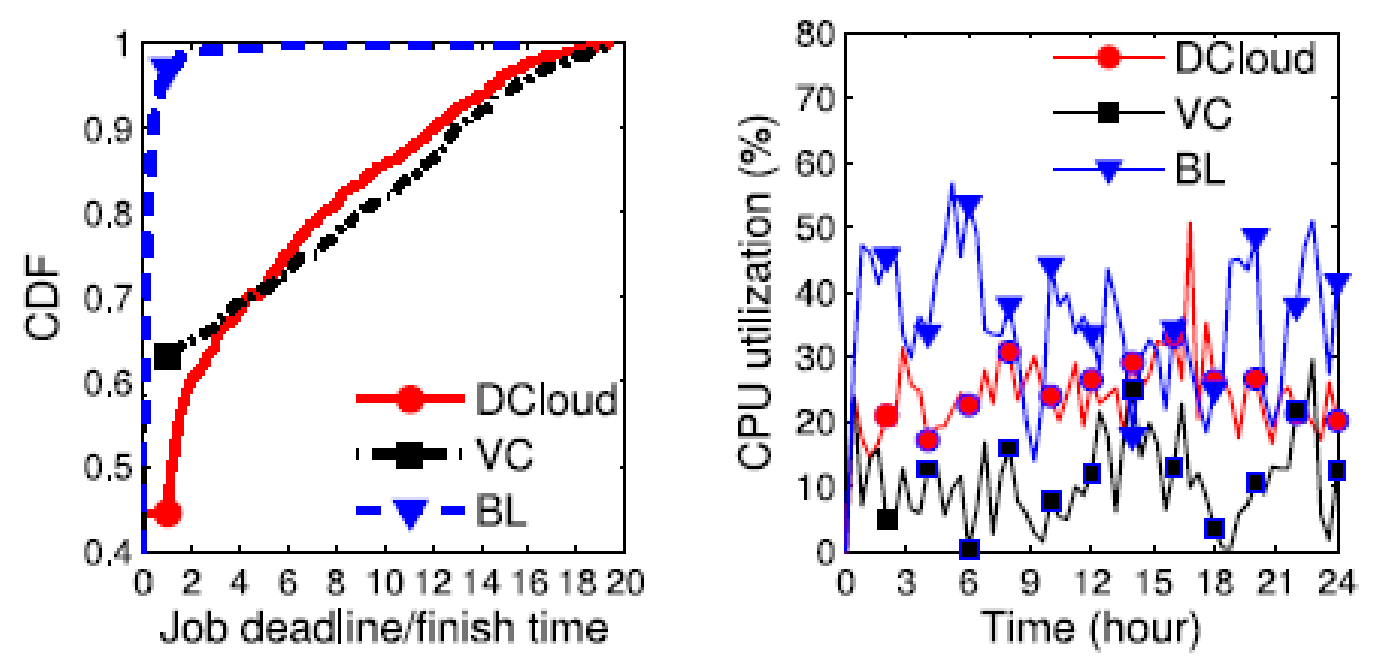

(a) CDF of jobs for the ratio of (b) Average CPU utilization over deadline over finish time time 


\section{CONCLUSION AND FEATURE WORKS}

In this mixed batch and transactional workloads for cloud computing jobs, it can meet the both the deadline cloud and resourcefully make use of the cloud computing source. By the demand of render to submit the both the needs of deadline and resource demand, this Deadline cloud and mixed batch allocation for transactional workloads for cloud job is provides the humdrum recital to application and then it foliage the space for seminal the reserve request to enhanced match the remaining resource. This deadline cloud computing uses the time gliding and bandwidth climbing to control the most suitable time intermission to begin each job, as well as the VM location and earmarked connection bandwidth. Because of this indicting process to persuade self-seeking renters to suggest the actual vital resource which make the algorithms works extra positively(resource allocation algorithm)Far-reaching simulations and test bed trials demonstrate that, likened with the baseline sharing and newly future VC allotment, in this deadline cloud and mixed batch and transactional workloads for cloud computing jobs can finish ominously more jobs within the deadline time, it makes the greater use of VM and system resources, for the gain and the extra profits.

\section{REFERENCES}

[1] Amazon Elastic Compute Cloud [Online]. Available: www.aws.amazon.com/ec2 Q2

[2] Google App Engine [Online]. Available: www.code.google. com/appengine/

[3] Windows Azure Platform [Online]. Available: ww.microsoft.com/windowsazure/

[4] H. Rodrigues, J. R. Santos, Y. Turner, P. Soares, and D. Guedes, "Gatekeeper: Supporting bandwidth guarantees for multi-tenant datacenter networks," in Proc. 3rd Conf. I/O Virtualization, 2011, p. 6.

[5] H. Ballani, P. Costa, T. Karagiannis, and A. Rowstron, "Towards predictable datacenter networks," in Proc. ACM SIGCOMM Conf., 2011, pp. 242-253.

[6] N. G. Duffield, P. Goyal, A. Greenberg, P. Mishra, K. Ramakrishnan, and J. E. Van der Merwe, "Resource management with hoses: Point-to-cloud services for virtual private networks," IEEE/ACM Trans. Netw., vol. 10, no. 5, pp. 679-692, Oct. 2002.

[7] D. Xie, N. Ding, Y. C. Hu, and R. Kompella, "The only constant is change: Incorporating Time-varying network reservations in data centers," in Proc. Conf. Appl., Technol., Archit., Protocols Comput. Commun., 2012, pp. 199-210.

[8] J. Zhu, D. Li, J. Wu, H. Liu, Y. Zhang, and J. Zhang, "Towards bandwidth guarantee in multi-tenancy cloud computing networks," in Proc. IEEE Int. Conf. Netw. Protocol, 2012, pp. 1-10.

[9] V. Jalaparti, H. Ballani, P. Costa, T. Karagiannis, and A. Rowstron, "Bridging the tenant-provider gap in cloud services," in Proc. $3^{\text {rd }}$ ACM Symp. Cloud Comput., 2012. pp 1-14.

[10] L. Popa, G. Kumar, M. Chowdhury, A. Krishnamurthy, S. Ratnasamy, and I. Stoica, "Faircloud: Sharing the network in cloud computing," in Proc. ACM SIGCOMM Conf. Appl., Technol., Archit., Protocols Comput. Commun., 2012, pp. 187-198.

[11] Time and cost optimization algorithm for scheduling multiple workflows in hybrid cloud BA Kumar, T Ravichandran European Journal of Scientific Research 89 (2), 265-275

[12] Scheduling Multiple Workflow Using De-De Dodging Algorithm and PBD Algorithm in Cloud: Detailed Study BA Kumar, T Ravichandran World Academy of Science, Engineering and Technology, International Journal.

[13] A Survey on Determining the Characteristics of Deadlock, Scheduling and Workflow Instances and Providing New Algorithms for the Issues Arising in Hybrid CloudB Arunkumar, $\mathrm{T}$ Ravichandran International Journal of Computer Applications 110 (14)

[14] Scheduling Multiple Workflow Using De-De Dodging Algorithm and PBD Algorithm in Cloud: Detailed Study BA Kumar, T Ravichandran World Academy of Science, Engineering and Technology, International Journal

[15] Task Scheduling and Seedblock Based Fault Tolerance in Cloud B Arunkumar, M Kesavamoorthi International Journal of Applied Engineering Research 11 (6), 4428-4432 